\title{
Characterizing Common Challenges Faced by Parental Caregivers of Children With Type 1 Diabetes Mellitus in Mainland China: A Qualitative Study
}

\section{Huijuan Tong}

Shengjing Hospital of China Medical University;Shenyang Medical college

\section{Feng Qiu}

Shenyang Fourth People's Hospital

Ling Fan ( $\square$ fanl_sj0512@163.com )

Shengjing Hospital of China Medical University https://orcid.org/0000-0001-5276-3921

\section{Research}

Keywords: Type 1 Diabetes Mellitus, Grounded Theory, Qualita tive Methods, Caregiv ing, Diabetes Care, Psychological Perceptions

Posted Date: November 23rd, 2020

DOI: https://doi.org/10.21203/rs.3.rs-111989/v1

License: @ (i) This work is licensed under a Creative Commons Attribution 4.0 International License.

Read Full License 


\section{Abstract}

Background: Parental caregiver psychology affects outcomes of children with Type 1 Diabetes Mellitus (T1DM). Objectives: To qualitatively examine perceptions of commo $\mathrm{n}$ challenges among parental caregivers of children with T1DM (T1DM Parental Caregivers).

Methods: 45-60 minutes semi structured interviews were conducted with T1DM Parental Caregivers at Shengjing Hospital, China Medical University form January 2018 to Jun e 2019. Interview recordings were transcribed and coded in NVivo 11.0 to observe emergent themes.

Results: Content analysis revealed 5 common themes with 14 subthemes, including persistent psychological stress (catastrophic emotions, emotional distress, a nd altered self efficacy), family function change (altered family life patterns and changes in parental role/function), challenges in daily management (technical challenges, emotional regulation, parent child conflict, and transition of care autonomy from parent to child), financial burden (cost burden of treatment and altered family economics), and lack of social support (social activity limitations and insufficient support systems). All were highly saturated across interview data.

Conclusions: Young children with T1DM rely on parental caregivers for disease management and to one day become capable of autonomous self management as they age. However, both the period of emotional disturbance during initial diagnosis, psychological stresses of long term care giving, and conflict emerging from transition for parental to child responsibility for care can cause psychological effects detrimental to parental caregivers and T1DM children. This work provides compelling evidence for the role of assessment and intervention in parental caregiver psychological and emotional wellbeing in diabetes care, as well as the need for improved social and school support for children with T1DM in China. school support for children with T1DM in China.

\section{Full Text}

This preprint is available for download as a PDF. 